\title{
$3 D$ imaging insights into forests and coral reefs
}

3 Kim Calders ${ }^{1,2, *}$, Stuart Phinn ${ }^{2}$, Renata Ferrari ${ }^{3}$, Javier Leon ${ }^{4}$, John Armston ${ }^{5}$, Gregory P.

4 Asner $^{6}$, Mathias Disney ${ }^{7,8}$

${ }^{1}$ CAVElab - Computational \& Applied Vegetation Ecology, Faculty of Bioscience Engineering, Ghent University, Belgium;

${ }^{2}$ Remote Sensing Research Centre, School of Earth and Environmental Sciences, University of Queensland, Brisbane 4072,

8 Australia;

$9{ }^{3}$ Australian Institute of Marine Sciences, Townsville, 4810, Australia;

$10{ }^{4}$ Global-Change Ecology Research Group, School of Science and Engineering, University of the Sunshine Coast, Sippy Downs,

11 4556, Queensland, Australia;

$12{ }^{5}$ Department of Geographical Sciences, University of Maryland, College Park, MD 20742, USA;

$13{ }^{6}$ Center for Global Discovery and Conservation Science, Arizona State University, Tempe, AZ 85281, USA;

$14 \quad{ }^{7}$ UCL Department of Geography, Gower Street, London WC1E 6BT, UK;

$15 \quad{ }^{8}$ NERC National Centre for Earth Observation (NCEO), UK

16 * correspondence: kim.calders@ugent.be ; @ kimcalders, @ stuartphinn, @DrFerrariR, @JXavierLeon, @ ArmstonJohn,

$17 @$ greg_asner, @mathiasdisney

19 Word count:

Abstract: 49/50

Main: 1198/1200

\section{ABSTRACT:}

24 Forests and coral reefs are structurally complex ecosystems threatened by climate change. In situ

$253 \mathrm{D}$ imaging measurements provide unprecedented, quantitative and detailed structural information

26 that allows testing of hypotheses relating form to function. This affords new insights into both individual organisms and their relationship to their surroundings and neighbours.

30 Corals and trees form the building blocks of their respective ecosystems. The structural complexity

31 of forests and coral reefs plays an important role in the biodiversity, productivity and functionality

32 of these ecosystems [1]. Corals and trees have been hypothesised to follow similar architectural 33 growth rules that shape their structures [2]. Structure and function are linked: organisms have 34 evolved under constraints of nutrients, light, water or space limitations and competition, and reproduction strategies, Box 1. Understanding 3D structure will assist in making links between 
36 structure and function that are needed to develop a general theory of ecosystem assembly and 37 function [3].

39 New in situ 3D structural measurements from terrestrial LiDAR and Structure-from-Motion (SfM) 40 analysis of digital photography enable precise, accurate and comprehensive structural 41 measurements. These measurements have already provided unique insights into forests and coral 42 reef ecosystems [3,4], but it is only now that analytical processing methods are sufficiently mature 43 to assist in understanding functional-structural relationships by testing hypotheses relating form 44 and function. Critically, these improved measurement approaches will allow more accurately defined baseline mapping and quantitative monitoring. When combined with traditional ecological and physiological knowledge, along with airborne or satellite remote sensing, such in situ observations can revolutionise how we monitor and manage forest and coral reef ecosystems in a 48 changing climate.

\section{In situ monitoring of 3D ecosystem structure}

51 Before the 1980s, the structure of ecosystems was often represented qualitatively and often only in 52 2D, e.g. via the hand-drawn architectural models of trees and corals [2]. Representing inherently 53 3D structural properties in 1D or 2D can help simplify analysis, but also implies a loss of 54 information of the ecosystem that may be vital for a proper understanding of key ecological services 55 [1]. Attempts to collect detailed 3D data were often invasive, such as the felling of trees or the use 56 of chain-and-tape methods in coral reefs.

58 Non-invasive in situ 3D imaging technologies either use direct measurements using LiDAR (Light

59 Detection and Ranging, also called laser scanning) or through indirect Structure-from-Motion 60 measurements derived from overlapping images (figure 1). SfM has existed since the late 1970s, 61 but advances in data collection via drones, autonomous underwater vehicles and even handheld 62 cameras have driven software developments, processing speed and robustness to extract 3D 63 information from the images [5,6]. LiDAR and SfM sensors have become significantly cheaper, 64 democratizing access to data. Further advancements in systematic surveys methods have removed 65 the need for high-end navigation and controls, enabling precise georeferencing and site revisits for 66 monitoring [7]. This resulted in new applications, such as quantifying precise loss of ecosystems 67 or habitat by acute disturbance events. New developments in terrestrial LiDAR techniques in the 68 last decade have led to new advances for the in situ measurement of individual tree and forest 
structure, Figure 1. Whereas the initial focus was on complementing and improving forest

70 inventories [8], it is only now that we see the use of 3D architecture as model-input to understand

71 e.g. wind damage in forests [9]. However, the relative novelty of such detailed 3D structural data means there is a long way to go to understand how best to unlock their full potential or make reliable comparisons at multiple sites [10]. Bottlenecks include reliable extraction of small structural elements (e.g. tips of tree branches), automation and robustness of methods and reliable point cloud classification. LiDAR applications for subsea fine-scale structural assessments of coral reefs are limited so far because of power requirements (water attenuates the LiDAR signal) and scattering from particles in the water (more data noise).

\section{Ecosystem structure and climate change}

80 Three-dimensional in situ information from forests and coral reefs could play a key role in 81 understanding how ecosystems are changing. First, basic structural measurements of a tree or a 82 coral colony are often used to quantify presence and provide population and demographic 83 parameters. Second, aggregated structural measurements provide a topological understanding of structure, which relates to their functioning. Third, the structure of trees and corals directly relates to habitat suitability and interactions with other species [4].

Ecosystem structure and climate are closely linked: changes in climate lead directly to physical changes in ecosystem structure and vice versa. For example, the 2010 Amazon drought event lead to $2.2 \mathrm{Pg}$ carbon committed emissions due to increased tree mortality, as well as subsequent impacts on forest composition and resilience [11]. Large trees are more vulnerable to droughts [12], but currently also have the largest uncertainties associated with their biomass estimates [8], making

92 it difficult to define optimal climate mitigation actions. Similarly, ocean warming has caused 93 global-scale bleaching and catastrophic mortality in corals.

\section{The way forward}

96 Extreme climate events are impacting forests and coral reefs. The complementarity between finescale 3D in situ data and measurements over larger areas will be a catalyst for advancing our understanding of ecosystem functioning. In situ measurements provide the finest detail, but

99 generally lack the spatial coverage that is required to understand ecosystem functioning: linking

100 "smaller-area-higher-detail" with "larger-area-lower-detail" data will assist in understanding local 101 ecosystem processes regionally and globally. 
103 3D data at regional scales, primarily from airborne LiDAR, are often already available but limited 104 due to low temporal frequency and uncertain in situ data. These regional datasets are often 105 commissioned by national mapping or environmental agencies for purposes other than forest or 106 coral reef monitoring, but can provide wall-to-wall spatial coverage (albeit aggregated over the 107 data acquisition time). Increased public availability of these data will help to rapidly upscale our 108 understanding of vulnerable ecosystems. Some countries already make such data available, but 109 often with limited or no metadata or quality information.

111 A range of new purpose-built space missions just launched or planned will estimate the structure 112 and carbon of forests at global scales, and new initiatives aim to create global coral reefs maps that 113 can serve as baseline maps to monitor short-term changes. The success of these missions will be 114 directly related to ground calibration in which advancements in 3D monitoring will be important.

115 To successfully combine observations across scale domains, a quality assessment framework will 116 be essential. Measurement uncertainty will propagate through the upscaling chain from individuals 117 to communities to global datasets. To understand the uncertainty of satellite estimates of 118 aboveground biomass, for example, requires quantified uncertainty of carbon storage at tree level, 119 as well as how these individual uncertainties aggregate at plot and continental scales. The 120 recognition of terrestrial laser scanning for measuring detailed 3D vegetation structure in the most 121 recent IPCC national greenhouse gas reporting guidelines is a step in the right direction for reaching 122 international standards on quantified uncertainties.

124 Most of the processing methods for fine-scale 3D data are modified from methods that were 125 originally designed for less spatially explicit data. A key question that remains is: are we using 126 algorithms that are fit-for-purpose to exploit the full potential of these in situ data? Data 127 measurement has matured significantly over the years, but active restoration approaches require a 128 close integration between management practices and research. The relative low-cost and ease of 129 data acquisition will allow further analysis of structure-function relationships with detailed 4D 130 data, with time being the $4^{\text {th }}$ dimension. Such approaches will improve our knowledge of forest 131 growth dynamics or coral reef recovery by combining detailed structural metrics with ecosystem 132 models. 


\section{REFERENCES}

138 [1] Burns J H R, et al. (2015) Integrating structure-from-motion photogrammetry with geospatial 139 software as a novel technique for quantifying 3D ecological characteristics of coral reefs. PeerJ $140 \quad 3: \mathrm{e} 1077$

141 [2] Dauget J M (1991) Application of tree architectural models to reef-coral growth forms. Mar 142 Biol 11:157-165

143 [3] Verbeeck H, et al. (2019) Time for a plant structural economics spectrum. Front For Glob 144 Change 2:43.

145 [4] Ferrari R., et al. (2018). Habitat structural complexity metrics improve predictions of fish 146 abundance and distribution. Ecography, 41(7), 1077-1091.

147 [5] Leon, J. X., et al. (2015) Measuring coral reef terrain roughness using 'Structure-from148 Motion' close-range photogrammetry. Geomorphology 242, 21-28.

149 [6] Smith, M.W., et al. (2015) Structure from motion photogrammetry in physical geography. 150 Prog. Phys. Geogr. 40, 247-275.

151 [7] Pizarro, O., et al. (2017). A simple, fast, and repeatable survey method for underwater visual 152 3D benthic mapping and monitoring. Ecol Evol 7, 1770-1782.

153 [8] Calders K, et al. (2015) Nondestructive estimates of above-ground biomass using terrestrial 154 laser scanning. Methods Ecol Evol 6:198-208.

155 [9] Jackson, T., et al. (2019) A New Architectural Perspective on Wind Damage in a Natural 156 Forest. Front For Glob Change 1:13.

157 [10] Duvall M S, et al. (2019). Collapsing Complexity: Quantifying Multiscale Properties of Reef 158 Topography. J Geophys Res-Oceans 124:5021-5038.

159 [11] Lewis S L, et al. (2011) The 2010 Amazon drought. Science 331:554-554.

160 [12] Bennett A, et al. (2015) Larger trees suffer most during drought in forests worldwide. Nat 161 Plants. 1:15139. 
Box 1: Definition of structural measurements.

Structure is the spatial arrangement of the basic components of a system. Within this context, structural measurements refer to both individuals (trees and corals), as well as their aggregated communities of forest and coral reef ecosystems. Structural measurements for trees include: tree height, canopy width, diameter at breast height, leaf and branch angle distribution and all aspects of biomass. These measurements can be made for individual trees as well as aggregate or average measurements at plot level. Structural measurements for corals include both colony height and width, while change in height per unit horizontal segment is termed rugosity or roughness. Rugosity is widely accepted as a measure of habitat complexity [1]. Structural parameters of forests and coral reefs are used in their baseline inventory and taxonomic mapping, and for regular field-based monitoring. The main advantages of terrestrial LiDAR and Structure-from-Motion measurements over traditional in situ manual measurements are their increased accuracy, precision, certainty, repeatability and traceability. Traceability is defined as the chain of steps taken from raw data to derived structural metric. This is an important process in establishing reliable baseline scenarios of structure with quantified uncertainties that are required to quantify structural change over time.

Figure 1: New in situ 3D technologies provide unprecedented insights into the place of individual trees and corals in forests and reefs.

(top) Left panel: A 3D render of Lagoon Reef, far northern Great Barrier Reef, Australia, derived from Structure-from-Motion algorithms applied to overlapping hand-held camera images collected

187 in the field [5]. Right panel: Deriving quantitative structural measurements from in situ 3D data.

188 Rugosity, or relative variation in height per unit distance (in $0.25 \mathrm{~m}$ pixels ) of Lagoon Reef, far 189 northern Great Barrier Reef, Australia.

190 (bottom) 3D complexity of a 1 hectare tropical savanna in Litchfield (Northern Territory, Australia) 191 captured using terrestrial LiDAR. Left panel: The colours represent canopy height (blue $=0 \mathrm{~m}$, red $192 \geq 25 \mathrm{~m}$ ). Right panel: Derived plant area volume density as a function of canopy height (in $1 \mathrm{~m}$ 193 height bins). This structural metric tells us how the volume of leaf and branch material is distributed 194 with height in the canopy. 


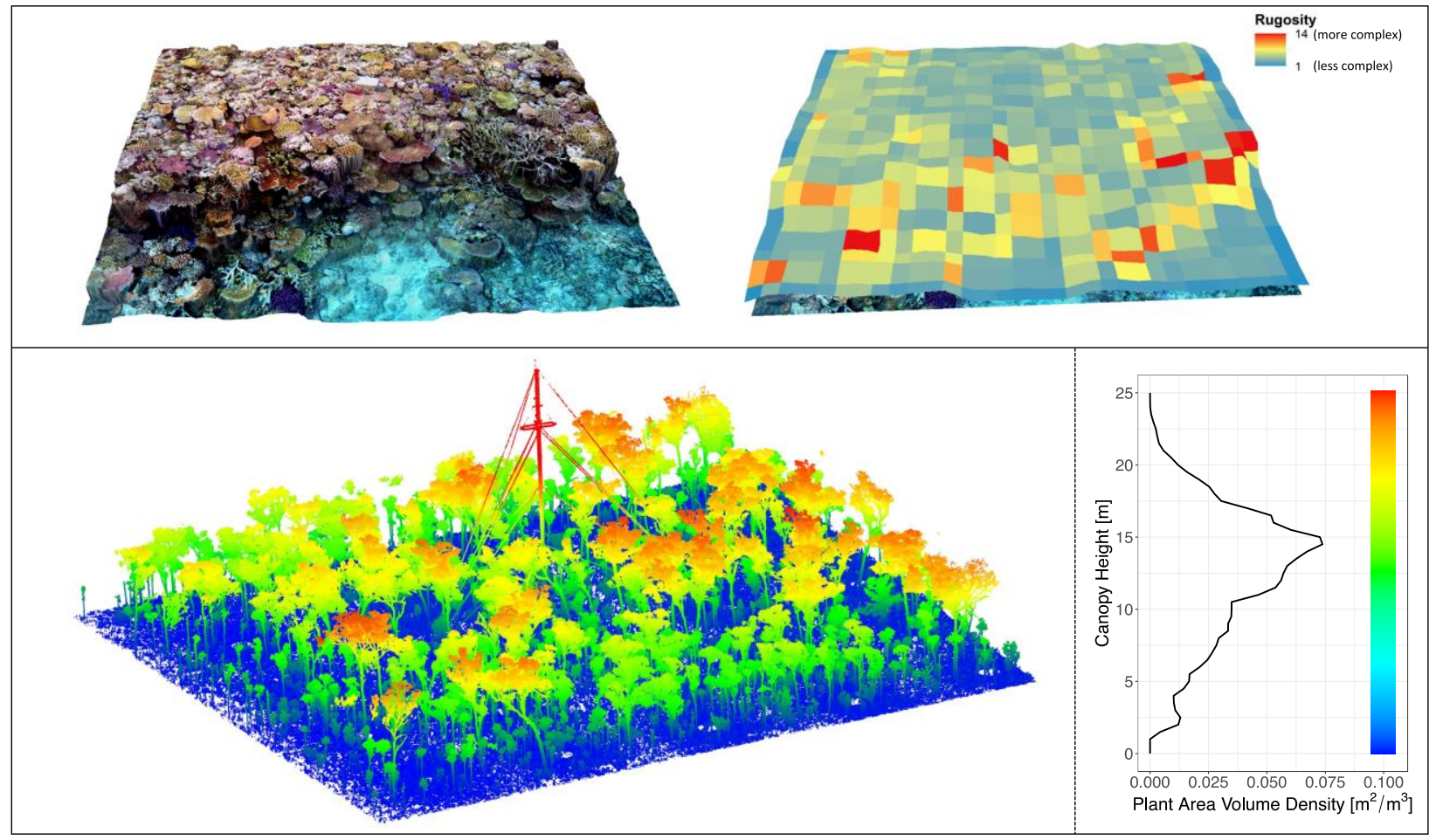

197 